\title{
Engaging Youth in a Mental Health System Improvement Initiative in Ontario: Developing the Be Safe Resource
}

\author{
Deanna Huggett, Andrea Flynn, and Alexia Jaouich \\ Centre for Addiction and Mental Health \\ Melissa Taylor-Gates and Scarlett Davidson \\ mindyourmind
}

\begin{abstract}
Youth engagement can improve the accessibility, effectiveness, and sustainability of programs and resources for youth. Little is known about youth engagement in system-level initiatives. As part of Systems Improvement through Service Collaboratives, an Ontario initiative that aimed to improve the system of care for youth with mental health or addictions needs, youth and adults in London, Ontario partnered to co-create Be Safe. The Be Safe resource helps youth manage mental health crises and identify local services. This article showcases a youth-adult partnership in a system-level initiative, and highlights a replicable strategy for developing resources relevant to youth needs and preferences.
\end{abstract}

Keywords: youth engagement, mobile app, mental health, addiction, system improvement, Ontario, implementation

\section{RÉSUMÉ}

La mobilisation des jeunes peut contribuer à améliorer l'accessibilité, l'efficacité et la durabilité des programmes et des ressources destinés à la jeunesse. Or, nous en savons très peu sur la mobilisation des jeunes dans les projets mis en place à l'échelle du système de santé mentale. Dans le cadre du projet

Deanna Huggett, Provincial System Support Program, Centre for Addiction and Mental Health, London, Ontario; Andrea Flynn, Provincial System Support Program, Centre for Addiction and Mental Health, London, Ontario; Melissa Taylor-Gates, mindyourmind, London, Ontario; Alexia Jaouich, Provincial System Support Program, Centre for Addiction and Mental Health, Toronto, Ontario; Scarlett Davidson, mindyourmind, London, Ontario.

The authors acknowledge the members of the London Service Collaborative as well as the youth and families with lived experience who openly partnered and contributed their insights to this system-level initiative, which included the development of the Be Safe application and resource toolkit.

Correspondence concerning this article should be addressed to Alexia Jaouich, Provincial System Support Program, Centre for Addiction and Mental Health, 33 Russell Street, Toronto, ON, M5S 2S1. Email: alexia.jaouich@camh.ca 
Améliorer les systèmes grâce aux projets de services axés sur la collaboration, créé en Ontario et visant à améliorer le système de soins pour les jeunes ayant des besoins reliés à des problèmes de santé mentale ou de toxicomanie, des jeunes et des adultes de London ont créé, en partenariat, une application mobile appelée Be Safe. Cette application aide les jeunes à gérer les crises en santé mentale et à trouver les ressources dont ils ont besoin. Dans cet article, les auteurs présentent ce partenariat créé par des jeunes et des adultes dans le cadre d'un projet mis en place à l'échelle du système de santé mentale, et ils montrent ainsi comment ce type de stratégie peut être reproduit pour permettre de concevoir et de mettre en place des ressources qui correspondent aux besoins et aux préférences des jeunes.

Mots clés : mobilisation des jeunes, application mobile, santé mentale, dépendance, amélioration du système de santé mentale, Ontario, mise en place.

Actively engaging individuals with lived experience ${ }^{1}$ of the mental health and addictions system is a critical and under-utilized element in many system-level initiatives (Mental Health Commission of Canada [MHCC], 2012). Both Changing Directions, Changing Lives: The Mental Health Strategy for Canada (hereafter the National Strategy, MHCC, 2012) and Open Minds, Healthy Minds: Ontario's Comprehensive Mental Health and Addictions Strategy (hereafter the Ontario Strategy, Ontario Ministry of Health and Long-Term Care [MOHLTC], 2011) highlight the need to more actively engage people affected by mental health challenges, including youth, in all aspects of system change.

Actively engaging individuals with lived experience involves more than simply seeking their feedback - it requires meaningfully collaborating with them to plan, organize, deliver, and evaluate services, resources, and supports (MHCC, 2012; MOHLTC, 2011). Existing literature confirms the many benefits of engaging youth as partners in planning services and problem-solving issues that affect them (Ontario Centre of Excellence for Child and Youth Mental Health, 2016; British Columbia Ministry of Children and Family Development [BC MCFD], 2013).

Much of this literature, however, focuses on the benefits of youth engagement for individuals and organizations. Less is known about youth engagement in system-level planning and implementation activities. The work of the London Service Collaborative in the Systems Improvement through Service Collaboratives (SISC, www.improvingsystems.ca) initiative provides an example of youth engagement as a key component of a community-based, system-level improvement project. SISC was an early initiative of the Ontario Strategy that brought together diverse cross-sector system stakeholders, including system and agency leaders, service providers, and individuals with lived experience, to address gaps in mental health and addictions systems in Ontario communities. Be Safe, developed as part of the London Service Collaborative's work, further illustrates how youth and adults can partner to co-create relevant, clinically sound resources for youth by combining their unique experiences and distinct expertise. To develop Be Safe, the London Service Collaborative partnered with mindyourmind, a national youth mental health program (www.mindyourmind. ca) based in London, Ontario. Be Safe is a mobile application (app) with a paper-based pocket guide companion designed to help youth manage mental health and addictions crises and identify appropriate supports based on their level of need. 
This article first provides a brief overview of the current literature highlighting the benefits of youth engagement. Subsequently, Be Safe is described, offering an example of a scalable system navigation and user empowerment tool. The youth-adult partnership applied in the London Service Collaborative is outlined to demonstrate how youth and adults can meaningfully partner to enrich decision making about system issues that affect youth. Finally, the co-creation process specifically involved in developing Be Safe is described to illustrate how youth engagement and youth-adult partnerships can facilitate the development of youthfriendly resources that are relevant to this population and informed by their personal expertise and experience. These examples of youth engagement speak to the National Strategy's priority of fostering collaboration at all levels, including meaningfully engaging individuals with lived experience, to help the system better meet the needs of people affected by mental health conditions.

\section{WHY ENGAGE YOUTH IN MENTAL HEALTH INITIATIVES?}

Mental health challenges affect many adolescents and young adults and influence their health throughout their lives (Public Health Agency of Canada, 2011). However, while an estimated 10-20\% of Canadian youth are affected by a mental illness or disorder, adolescents and young adults often do not seek and/or receive help for their mental health challenges (Canadian Institute for Health Information, 2015; Pearson, Janz, \& Ali, 2013; Gulliver, Griffiths, \& Christensen, 2010). In Ontario, recent data indicate that $17 \%$ of students aged 12 to 18 rate their mental health as fair or poor, and 28\% report they want to seek help for a mental health problem but do not know where to turn (Boak, Hamilton, Adlaf, Henderson, \& Mann, 2016). This evidence suggests an important disparity - namely, there is a clear need to support young people's mental health and well-being, yet a paucity of high-quality data on youth perceptions of barriers and facilitators to help-seeking (Ontario Centre of Excellence for Child and Youth Mental Health, 2016; Gulliver, Griffiths, \& Christensen, 2010).

Given that $70 \%$ of mental health problems have their onset during childhood or adolescence (Government of Canada, 2006), the mental health of children and youth is an identified priority in both the National and Ontario Strategies. The Strategies also encourage mental health service providers, planners, and funders to work with users of the system to identify how mental health systems can better address people's needs and foster recovery. To this end, youth engagement can provide important insights into why young people are not accessing mental health services, and thus can create opportunities to develop approaches and tools to better meet their needs. It can empower youth and encourage them to apply their knowledge and experience to shape mental health services and resources. In turn, this can improve the relevance and accessibility of supports geared toward youth (Ontario Centre of Excellence for Child and Youth Mental Health, 2016).

Evidence demonstrates that youth engagement in program planning has benefits at individual and organizational levels. At the individual level, youth can gain a sense of empowerment and form healthier relationships with peers and adults during, and following, participation in youth engagement activities. They can also develop leadership and problem-solving skills, and build capacities that support their transition to adulthood and independence (BC MCFD, 2013; Ontario Centre of Excellence for Child and Youth Mental Health, 2016). 
By interacting with youth as thought partners and collaborators, adults can gain a greater understanding of youth needs, strengths, and skills and learn new ways of approaching problems (BC MCFD, 2013; Ontario Centre of Excellence for Child and Youth Mental Health, 2016). For organizations, the benefits of youth-adult partnerships can include improved programmatic decision making around youth needs, greater accountability and transparency, and improved relationships with youth (BC MCFD, 2013; Ontario Centre of Excellence for Child and Youth Mental Health, 2016).

Overall, the existing literature highlights that actively engaging youth, and including them as partners in program planning and the decision-making processes, can create a supportive environment that encourages youth to contribute their knowledge as experts in their own needs and experiences. The process is enhanced by their energy, creativity, and passion for change (Halsall, Garinger, \& Forneris, 2014). Working with adult partners can enable youth to propel decision-making groups to greater innovation and productivity and facilitate better health outcomes for youth partners (Ontario Centre of Excellence for Child and Youth Mental Health, 2016).

The Be Safe resource and the process used in its development serves as an ideal case exemplar that aligns with the above evidence on youth engagement as well as National Strategy recommendations.

\section{THE BE SAFE RESOURCE}

Be Safe is a system navigation, empowerment, and support tool that was co-created through a youthadult partnership to help youth with mental health and addictions concerns gain control in addressing their own needs. Be Safe includes both a mobile app and a paper-based pocket guide companion. The mobile app makes effective use of technology to empower youth to find help when they need it, and supports them through the process of reaching out.

The app consists of several key elements that collectively provide a comprehensive tool to support youth. It specifically includes:

1. A customized safety plan, which youth are encouraged to complete when they are not in crisis and with the assistance of a trusted person in their support network (e.g., service provider, family member, friend, etc.).

2. A decision aid to support identification of appropriate community resources based on level of need at any given point in time. Youth provide responses in real time to a series of guided questions (e.g., about current emotions and level of safety); the output that is generated by the app provides relevant details for suggested services or supports, such as agency contact information, to help users access these resources.

3. A comprehensive listing of mental health services and supports in the community.

4. A personalized "Get Help" script, generated from data provided by users (e.g., name, specific concerns, medications, etc.) to facilitate their communication with professionals and other supports at times of crisis.

5. Information on how to wait safely for help, what youth can expect when they access resources, and youth rights as a client. 
The Be Safe app is available for free download at the Apple and Google Play stores, and accompanying resources are available at www.mindyourmind.ca and www.besafeapp.ca. The app is easy to use and available at any time to support youth. It has a clean interface, uses clear language, and employs a colour design that can be seen by individuals with colour blindness.

The companion paper version of Be Safe ensures that youth who do not have mobile devices can benefit from the resource. It can serve as a back-up copy that youth can keep in a safe location or give to a trusted person. It can also be easily integrated into service delivery processes. For example, service providers involved in the London Service Collaborative used the paper version with youth in hospital in-patient settings and youth justice programs by supporting them to complete the Be Safe safety plan with a clinician before discharge. Youth were encouraged to subsequently enter their safety plan into the app if they had access to a mobile device.

\section{Developing Be Safe as part of Systems Improvement through Service Collaboratives}

As part of the Ontario Strategy, SISC was tasked with improving the coordination of, and access to, mental health and addictions services. To accomplish this goal, 18 service collaboratives were established in communities across Ontario between 2011 and 2014. Collaboratives brought together diverse stakeholders to identify a local system need and implement an action plan to address this challenge. Individuals with lived experience were integral to the SISC initiative. Reflecting the Ontario Strategy's early focus on children and youth, older adolescents and young adults were active participants in SISC. They acted as Collaborative members in many communities and also served in various advisory and consultative capacities throughout the initiative.

SISC was supported by six provincial ministries ${ }^{2}$ and funded by the Government of Ontario. Collaboratives were locally led and community owned, and received implementation support from the Centre for Addiction and Mental Health's (CAMH) Provincial System Support Program (PSSP). CAMH is Canada's largest mental health and addictions teaching hospital, as well as one of the world's leading research centres in mental health and addictions. CAMH's PSSP was selected by the Ontario government to act as the SISC sponsor and serve as the "backbone organization" for the initiative - that is, an external entity providing support and coordination across all participating organizations and communities (Kania \& Kramer, 2011). With implementation teams situated in nine regional offices across Ontario and a central office in Toronto, PSSP works with partners across sectors to move evidence to action and transform mental health and addictions systems in Ontario. To accomplish this goal, PSSP applies expertise in knowledge exchange, implementation, evaluation, information management, and health equity and priority population engagement.

To guide the work of the collaboratives, PSSP's implementation teams used an evidence-based approach rooted in implementation science (specifically, the National Implementation Research Network's Active Implementation Frameworks, http://nirn.fpg.unc.edu/learn-implementation). Implementation science aims

to understand the behaviour of healthcare stakeholders and to identify and address the barriers to effective and sustainable adoption of evidence-informed interventions (National Institutes of Health, 2017). To support the collaboratives, PSSP implementation teams also applied other approaches and tools as appropriate, including those pertaining to quality improvement (e.g., plan-do-study-act cycles; Health Quality Ontario, 
2012), system design (e.g., client journey mapping; McCarthy et al., 2016), and health equity (i.e., the Health Equity Impact Assessment, Ontario; MOHLTC, 2012).

\section{Youth Engagement in the London Service Collaborative}

Engaging youth as partners in the London Collaborative facilitated the process, success, and sustainability of the Collaborative's work and prompted the development of the Be Safe resource. Four of the Collaborative's 32 members were youth. As full, active Collaborative members, these youth engaged with adult service providers and system leaders from mental health and addictions, education, child welfare, justice, and culturally specific (Francophone; First Nations, Métis, and Inuit) service sectors, among others. Youth Collaborative members contributed their creativity, culturally relevant strengths and skills, knowledge of youth needs, and experience of the system.

Youth were initially recruited to participate in the London Collaborative through service provider networks. In line with best evidence for engaging individuals with lived experience (Ontario Centre of Excellence for Child and Youth Mental Health, 2016; The Change Foundation, 2015), youth received monetary compensation for their time and were invited to bring members of their support networks to Collaborative meetings. Additional efforts were taken to support youth participation by hosting meetings in safe and accessible locations, providing refreshments, and arranging transportation as needed.

Youth were involved at all stages of the London Collaborative's work, beginning with identification of a system gap and selection of an intervention to address this gap. Over the course of several months, youth and adult Collaborative members engaged in various system mapping, needs identification, and gap prioritization activities. Through this exploration work, they identified a need to improve care for youth who present at the emergency department yet would be best served by community-based supports (rather than, for example, in-patient psychiatric care). Drawing on available evidence (including research, professional, and lived experience, and cultural/traditional knowledge), the Collaborative co-created an intervention that involved two key components:

1. A protocol to ensure smooth referrals from a local paediatric emergency department to community mental health intake services. This protocol facilitates continuity of care by formalizing pathways for hospital-to-community service transitions and outlining consistent procedures for sharing essential information across organizations. In their role as Collaborative members, youth contributed to decisions that informed the protocol.

2. The Be Safe resource, which, as described above, helps youth learn about services in the community, manage mental health and addictions crises, and access appropriate services based on their specific needs at a given point in time. The value of this resource emerged through discussions amongst youth and adult Collaborative members, who noted that youth sometimes present at the emergency department because they are unsure of other local resources available to support their needs. Be Safe responds to this issue by helping youth determine if there are resources other than the emergency department that they can reach out to for support.

Recognizing that engaging youth in the development of Be Safe would ensure that the resource was relevant and likely to be used by youth, the Collaborative approached and partnered with mindyourmind, 
which is a non-profit mental health program that co-develops innovative resources for youth, emerging adults, and the professionals who serve them. Consistent with their work on other initiatives, mindyourmind helped build understanding of, and capacity for, youth engagement; facilitated relationship development between youth and adults; assisted Collaborative members to gain perspective about their partners' views and cultures; and provided support and guidance to youth members of the Collaborative (Garinger et al., 2016).

To establish a shared understanding about the goals of the resource, initial conversations took place between youth and adult members of the Collaborative, staff and youth partners from mindyourmind, and the PSSP Implementation Team supporting the Collaborative. mindyourmind recruited additional youth participants from their youth partner network to support the development of the proposed resource. All of the youth involved in the initiative introduced the potential value of specifically developing a mobile app. From there, a co-creation process, described below, was launched to develop Be Safe.

\section{Youth Partners as Leaders in the Development and Dissemination of Be Safe}

To develop Be Safe, mindyourmind supported youth members of the Collaborative and additional youth to participate in a weekend-long intensive development session known as a "design studio." A design studio is a concentrated creation period in which stakeholders who will be the ultimate users of the product are engaged in the product's development. Participants are guided through an iterative process to identify and define an issue, and to generate prototype solutions as a collective. Both the solutions and the process are recorded, reflected upon, and evaluated (Duncan \& Breslin, 2009; Freire \& Sangiorgi, 2010; mindyourmind, 2015). Nine youth and young adults participated in the Be Safe design studio. By the end of the weekend, they had developed a preliminary draft of the key elements of the mobile app and paper-based pocket guide companion that would ultimately form Be Safe. Following the design studio, youth partners and staff at mindyourmind collaborated to refine the content and format of the resource in ongoing consultation with members of the London Collaborative and the PSSP Implementation Team.

Youth took a leadership role in gathering relevant information for the app (e.g., compiling detailed service listings across the London-Middlesex region). They also reached out to community clinicians to ensure that Be Safe provided accurate details about local resources and was aligned with evidence-based practices. Youth also built capacity among adult Collaborative members by sharing their expertise on how to best use technology to reach youth and how to engage youth to use the resource. Further, they supported planning for, and were actively engaged in, disseminating the resource.

Be Safe was widely disseminated to service providers for use with youth in diverse sectors of the London community, including hospital settings, community mental health and addictions agencies, school boards, and youth justice programs. It was broadly shared with stakeholders across Ontario through PSSP's Evidence Exchange Network (EENet, www.eenet.ca), a mental health and addictions knowledge exchange network. In addition, a provincial webinar and conference presentations on Be Safe were co-developed and facilitated by youth and adult partners from mindyourmind and PSSP.

Overall, Be Safe represents a system navigation tool that makes effective use of technology to empower youth to find help when they need it, and supports them through the process of reaching out. In alignment with the National Strategy, this resource provides an example of how a collaborative process that fosters the 
leadership of youth and individuals with lived experience can contribute to addressing the needs of young people living with mental health conditions.

\section{Success of the Youth-Adult Partnership Model}

A youth focus group and a survey of youth and adult London Collaborative partners were conducted to evaluate the experience of the youth-adult partnership and the process of developing Be Safe as part of the Collaborative's intervention. Eight youths who were involved in developing Be Safe, including the four youth Collaborative members, participated in a focus group. Six of these youths also completed a questionnaire about their partnership experience, adapted from The Youth Engagement Toolkit (BC MCFD, 2013). Both the focus group and the survey invited the youths to reflect on their experiences with the Collaborative, as well as on the development of Be Safe. Feedback on the youth-adult partnership from the adult perspective was gathered using a survey (also adapted from The Youth Engagement Toolkit; BC MCFD, 2013). This survey was completed by nine adult Collaborative members.

Consistent with youth engagement literature on effective youth-adult partnerships (BC MCFD, 2013; Ontario Centre of Excellence for Child and Youth Mental Health, 2016), both youth and adult Collaborative members described youth as equal partners in decision making and important contributors to the process, and viewed the engagement as meaningful for all individuals and organizations involved.

Youth emphasized that they felt appreciated by adult partners and had a positive learning experience as a result of their involvement with the Collaborative:

I grew a ton from [being part of the Service Collaborative] and I'm so thankful.

People acknowledged me. I was on equal footing.

As a member of the London Service Collaborative, I learned a lot about making change at the system level.

Adults articulated the unique contributions that youth made to the project and highlighted the broader importance of youth engagement in program and system change initiatives:

The youth brought a very progressive way of thinking and great creativity to the project.

[The youth] perspective and energy was incredible. To see their level of empowerment and ownership of the work to be completed is energizing, and instills a strong sense of commitment in myself to make the system work better for them.

In order to be relevant, we need [youth] at all of our tables ... planning, development, implementation, and evaluation.

Youth and adults also identified lessons learned and recommended strategies to improve future youthadult partnerships. Youth indicated that additional education for both youth and adults about effective youthadult partnerships would make the collaboration even more productive and enjoyable. Adults recognized that they had a role to play in actively creating opportunities for youth participation throughout the project, and acknowledged room for improvement in this capacity. Youth noted that Collaborative meetings were at times bureaucratic and dry, while adult partners noted some uncertainty about how best to approach situations where youth and adult perspectives did not align. Nevertheless, both youth and adult partners generally 
affirmed the value of the partnership, with one youth specifically commenting, "the pros of being involved [in the youth-adult partnership] outweighed the cons."

Surveys and focus groups further demonstrated the beneficial impact of the partnership on the innovative design and relevance of Be Safe:

Having youth with lived experience really shaped and influenced the final product of the Be Safe app. This was essential in creating a product that would really resonate with the intended audience-youth. (adult)

This participant's comment is further evidenced by the success of Be Safe and the subsequent spread of this resource to additional communities.

\section{Success of Be Safe}

When first introduced by the London Collaborative in 2014, the Be Safe resource list focused on organizations and programs specific to the London-Middlesex community. Widespread knowledge exchange sparked interest about Be Safe across the province, supporting scaling of the resource and facilitating updates and improvements to the mobile app. Originally, Be Safe was available only through mindyourmind's website; however, increased demand and expansion to additional communities resulted in the development of a dedicated website, www.besafeapp.ca. In addition, the resource has been translated into French and Arabic.

As of March 2017, mindyourmind has supported 30 Ontario communities to adapt Be Safe to their local context. For a nominal fee, mindyourmind works with community partners to collect information about local resources for youth, and manages the development of the app directly. Communities that have not commissioned a local adaptation can access a version of Be Safe that lists provincial-level resources.

The process of adapting Be Safe and the model of youth engagement used in its development have also facilitated other opportunities for relationship building and innovation within Ontario communities. For example, while working on developing a local service listing for Be Safe, community partners in Hamilton, Ontario uncovered gaps in local crisis planning for youth. They proceeded to map existing crisis planning mechanisms and sought to develop more proactive ways of supporting youth in crisis. This included implementing Be Safe as the standard crisis-planning tool for the largest child mental health agency in the Hamilton area and the local team of child and youth crisis service providers.

Be Safe has garnered attention from organizations and communities across Canada. As of March 2017, there have been more than 11,140 Be Safe sessions ${ }^{3}$ by users in Canada, including 2,234 sessions (8.5\%) from locations outside Ontario. Further, the "How to Use the Be Safe Resource" YouTube video has been viewed more than 2,234 times. mindyourmind has also received expressions of interest to facilitate youthadult partnerships and adapt $\mathrm{Be}$ Safe to communities outside of Ontario, including from locations in Western Canada and the United States.

\section{CONCLUSION}

This article describes how youth with lived experience in London, Ontario actively contributed to a system-level improvement initiative. The direct involvement of youth in the planning, implementation and decision-making processes of the London Service Collaborative demonstrates that youth and adults can 
meaningfully partner and contribute unique perspectives to support system-level initiatives. Further, the co-creation model specifically used to develop Be Safe provides an example of a youth engagement strategy that can be easily replicated to develop resources that are relevant to youth needs and preferences.

Be Safe itself is an example of an innovative self-management and system navigation tool that is being scaled to additional Ontario communities with the goal of empowering more youth with mental health challenges to find and access the right supports where and when they are needed. Both the processes and the resource described in this article represent replicable opportunities to enhance youth-adult collaboration in the context of mental health system improvement, thus providing examples of community-based efforts that align with National Strategy priorities and hold potential for further growth and impact.

\section{ENDNOTES}

1. For the purposes of this article, individuals with lived experience include those with experience in the mental health and addictions system as clients, family members, and/or supporters of clients.

2. Ministry of Health and Long-Term Care; Ministry of Children and Youth Services; Ministry of Education; Ministry of Training, Colleges and Universities; Ministry of the Attorney General; and Ministry of Community Safety and Correctional Services.

3. A session is a term used by Google Analytics to define a group of hits recorded for a user in a given time period. For example, in a single session, a user can view multiple screens or pages on the app (https://support.google. com/analytics/answer/2731565?hl=en)

\section{REFERENCES}

Boak, A., Hamilton, H. A., Adlaf, E. M., Henderson, J. L., \& Mann, R. E. (2016). The mental health and wellbeing of Ontario students, 1991-2015: Detailed OSDUHS findings (CAMH Research Document Series No. 43). Toronto, ON: Centre for Addiction and Mental Health. Retrieved from http://www.camh.ca/en/research/ news_and_publications/ontario-student-drug-use-and-health-survey/Documents/2015\%20OSDUHS $\% 20$ Documents/2015OSDUHS_Detailed\%20MentalHealthReport.pdf

British Columbia Ministry of Children and Family Development. (2013). Youth Engagement Toolkit Resource Guide. Retrieved from http://www.mcf.gov.bc.ca/youth_engagement/pdf/yet_resource_guide.pdf

Canadian Institute for Health Information. (2015). Care for Children and Youth with Mental Disorders. Retrieved from https://secure.cihi.ca/free_products/CIHI\%20CYMH\%20Final\%20for\%20pubs_EN_web.pdf

Duncan, A. K., \& Breslin, M. A. (2009). Innovating health care delivery: The design of health services. Journal of Business Strategy, 30(2/3), 13-20. doi:10.1108/02756660910942427

Freire, K., \& Sangiorgi, D. (2010). Service design \& healthcare innovation: From consumption to co-production and co-creation. Second Nordic Conference on Service Design and Service Innovation. Retrieved from http://www. servdes.org/pdf/freire-sangiorgi.pdf

Garinger, C., Reynolds, K., Walker, J., Firsten-Kaufman, E., Raimundo, A., Fogarty, P., Leonhart, M., \& Mobilizing Minds Research Group. (2016). Mobilizing minds: Integrated knowledge translation and youth engagement in the development of mental health information resources. Gateways: International Journal of Community Research and Engagement, 9(1), 172-185. doi:10.5130/ijcre.v9i1.4415

Government of Canada. (2006). The human face of mental health and mental illness in Canada. Retrieved from http:// www.phac-aspc.gc.ca/publicat/human-humain06/pdf/human_face_e.pdf

Gulliver, A., Griffiths, K. M., \& Christensen, H. (2010). Perceived barriers and facilitators to mental health help-seeking in young people: A systematic review. BMC Psychiatry, 10, 113. doi:10.1186/1471-244X-10-113

Halsall, T., Garinger, C., \& Forneris, T. (2014). mindyourmind: An overview and evaluation of a web-facilitated mental health program that applies empowerment strategies for youth. Journal of Consumer Health on the Internet, 18(4), 337-356. doi:10.1080/15398285.2014.952998 
Health Quality Ontario. (2012). Implementing rapid cycle improvements: What are plan-do-study-act (PDSA) cycles? In Quality Improvement Guide (section 3.6). Retrieved from http://www.hqontario.ca/portals/0/documents/qi/ rf-document-pdsa-cycles1-en.pdf

Kania, J., \& Kramer, M. (2011). Collective Impact. Stanford Social Innovation Review. Retrieved from https://ssir.org/ images/articles/2011_WI_Feature_Kania.pdf

McCarthy, S., O'Raghallaigh, P., Woodworth, S., Lin Lim, Y., Kenny, L. C., \& Adam, F. (2016). An integrated patient journey mapping tool for embedding quality in healthcare service reform. Journal of Decision Systems, 25(sup1), 354-368, doi:10.1080/12460125.2016.1187394

Mental Health Commission of Canada. (2012). Changing directions, changing lives: The mental health strategy for Canada. Retrieved from http://strategy.mentalhealthcommission.ca/pdf/strategy-images-en.pdf

mindyourmind. (2015). (Presentation). Youth Engagement and Design. Retrieved from https://prezi.com/6ixraw6xa730/ mindyourmind-youth-engagement-and-design/

National Institutes of Health, Fogarty International Center. (2017, April 5). Implementation science information and resources. Retrieved from: https://www.fic.nih.gov/researchtopics/pages/implementationscience.aspx

Ontario Centre of Excellence for Child and Youth Mental Health. (2016). Walking the talk: A toolkit for engaging youth in mental health. Retrieved from http://www.yetoolkit.ca

Ontario Ministry of Health and Long-Term Care. (2011). Open minds, healthy minds: Ontario's comprehensive mental health and addictions strategy. Retrieved from http://www.health.gov.on.ca/en/common/ministry/publications/ reports/mental_health2011/mentalhealth_rep2011.pdf

Ontario Ministry of Health and Long-Term Care. (2012). Health Equity Impact Assessment (HEIA). Retrieved from http://www.health.gov.on.ca/en/pro/programs/heia/

Pearson, C., Janz, T., \& Ali, J. (2013). Health at a glance: Mental and substance use disorders in Canada. Statistics Canada. Catalogue no. 82-624-X.

Public Health Agency of Canada. (2011). The chief public health officer's report on the state of public health in Canada: 2011 youth and young adults - life in transition. Retrieved from http://www.phac-aspc.gc.ca/cphorsphcrespcacsp/2011/cphorsphc-respcacsp-06-eng.php

The Change Foundation. (2015). Should money come into it? A tool for deciding whether to pay patient-engagement participants. Retrieved from http://www.changefoundation.ca/patient-compensation-report/ 